\title{
Involuntary occupational drug exposure
}

\author{
Frederic J. Deschamps* and Audrey Cecile-Bartelt \\ Department of Occupational Health, Medicine Faculty, Reims, France
}

Addiction by inhalation is a problem becoming more and more usual all around the world. Thus, the drug inhalation exposure can cause a lot of health tragedies. The origin of these diseases could be due to acute or chronic mixed drug inhalations. Moreover, most of the clinical signs observed are not specific. The major complication of drug inhalation is either sudden sniffing death syndrome, or cardiomyopathy, or central nervous system toxicity, or haematological abnormalities, or kidney and hepatocellular toxicities. There are various inhalants composed of different ingredients, which are used for various purposes such as the storage of teargas grenades - the ones used during riots. This particular case of intoxication endured by a gunsmith while sniffing volatile substance during work is an involuntary drug abuse.

In order to illustrate this kind of scenario, we are presenting a case report about teargas usually used in riots. Actually, teargas is not a gas but a toxic chemical irritant in the form of powder or drops mixed in variable concentrations ( 1 to $5 \%$ ) with a solvent, and delivered by a dispersion device. The five chemical components traditionally used in the E.U. are Chlorobenzylidene-malonanitrile (also known as $\mathrm{CS}$ ), Chloracetophenone (CN or "mace"), dibenzoxazepine (CR), oleoresincapiscum (OC), and pelargonic acid vanillylamide (PAVA). But still, involuntary drug exposure during work is not usual.

$\mathrm{Mr} \mathrm{H}$. consulted a doctor in an occupational diseases department, after an occupational accident that occurred two weeks ago. He was a gunsmith employed by a French national police school. The day before, $\mathrm{Mr} \mathrm{H}$. received a teargas grenades delivery from another training centre. He noticed that they were leaking, and three days later, his arms and face itched; he also complained about memory disorders. Six days after, he suffered from dizziness and asthenia, which were followed by nausea, vomiting, shivering and sweating. Therefore, $\mathrm{Mr} \mathrm{H}$. transferred the grenades away from his workplace and consulted an ENT specialist who concluded that his dizziness has no specific ENT pathology implications. Eight days after the beginning of the exposure, he stopped working. The symptoms partially faded. A month after the exposure, the magnetic resonance imagery (MRI) and the Electroencephalography (EEG) were normal. In spite of everything, he continued suffering headaches and equilibrium disorder; he also lost weight. A year after the incident, he still suffers from recurrent headaches knowing that he did not have any migraine criteria.

In medical literature on CS, the focus is mainly on acute irritant effects and concerns transient dermal and ocular injuries. In literature, no case report of chronic exposition to chlorobenzilidenemalononitrile was found. The ocular effects were mainly as irritating as blepharospasm, lacrimation, and conjunctivitis are. However, CS could rarely cause corneal epithelial damage. Respiratory effects reported are sore throat and chest pain, but only one case report states moderate outbreaks of recurrent cough and wheezing consistent with airways dysfunctional syndrome [1]. Another case report states identified pulmonary oedema [2]. Concerning the dermal effects, several case reports mentioned first and second degree burns [3]. An author also reports multisystem hypersensitivity reactions with erythroderma, hepatitis, wheezing, pneumonitis and hypereosinophilia [1]. In literature, other symptoms are mentioned such as headaches, vomiting, haemoptysis and haematemesis [4]. In our case, few symptoms are found in other publications (such as vomiting, headaches and dermal irritation), but dizziness, asthenia and weight-loss are not references. Moreover and according to our case, the patient did not show lachrymal irritations during and after contact. He was exposed to a low quantity of CS during a long period. Therefore, low level and long duration of exposure may explain the fact that ocular symptoms were absent. Also, equilibrium disorders in relationship with cochlear disturbances may be explained by animal experimentation (on rats) [5]. Then, a toxic effect of few metabolites has been documented in animal experimental studies, in particular with chlorobenzylidene malononitrile (formation of cyanide and thiosulfate derivatives) and chloracetophenone (formation of hydrogen chloride). CS is metabolized to o-chlorobenzyl malononitrile (CS H2), o-chlorobenzaldehyde, o-chlorohippuric acid, and thiocyanate. Thiocyanate was found in both urine of a group of exposed animals and men, after an inhalation exposure. Hydrogen cyanide (undiscounted acid) prevents the use of oxygen by blocking the electron transfer from cytochrome a3 to molecular oxygen. That means that it blocks the respiration of the cells or the oxidative metabolism even if the particular pressure of oxygen in the tissue is normal. Cells of brain are indeed very sensitive to the effects of $\mathrm{HCN}$. The evidence for the endogenous release of cyanide in rats inhaling CS is based on increased urinary excretion of thiocyanate [6]. Besides, it seems important to provide a short recap of the CS metabolism. CS is hydrolysed with the production of malononitrile and 2-chlorobenzaldehyde (which is secondly transformed in 2-chlorohippuric acid) [7].

In conclusion, this case report displays medical symptoms in relation with chronic exposure to low levels of teargas. The characteristics of this exposure are unusual, because usually, teargas is used to provoke acute effects during a short period. Thus, this occupational case can be studied in comparison to involuntary inhalation causing a drug abuse.

\section{References}

1. Hill AR, Silverberg NB, Mayorga D, Baldwin HE (2000) Medical hazards of the teargas CS. A case of persistent, multisystem, hypersensitivity reaction and review of the literature. Medicine (Baltimore) 2000; 79:234-40. [Crossref]

2. Krapf R, Thalmann H (1981) [Acute exposure to CS tear gas and clinical studies]. Schweiz Med Wochenschr 111: 2056-2060. [Crossref]

Correspondence to: Frederic J. Deschamps, Department of Occupational Health, Faculté de Médecine, 51 rue Cognacq-Jay, 51100, Reims, France; E-mail: fdeschamps@chu-reims.fr

Received: December 29, 2015; Accepted: January 25, 2016; Published: January 28, 2016 
3. Zekri AM, King WW, Yeung R, Taylor WR (1995) Acute mass burns caused by o-chlorobenzylidene malononitrile (CS) tear gas. Burns 21: 586-589. [Crossref]

4. Anderson PJ, Lau GS, Taylor WR, Critchley JA (1996) Acute effects of the potent lacrimator o-chlorobenzylidene malononitrile (CS) tear gas. Hum Exp Toxicol 15: 461465. [Crossref]

5. Fechter LD, Klis SF, Shirwany NA, Moore TG, Rau DB (2003) Acrylonitrile produces transient cochlear function loss and potentiates permanent noise - induced hearing loss. Toxicol Sci 75:117. [Crossref]

6. Frankenberg L, Sörbo B (1973) Formation of cyanide from o-chlorobenzyliden malononitrile and its toxicological significance. Arch Toxikol 31: 99-108. [Crossref]

7. Brewster K, Harrison JM, Leadbeater L, Newman J, Upshall DG (1987) The fate of 2-chlorobenzylidene malononitrile (CS) in rats. Xenobiotica 17: 911-924. [Crossref]

Copyright: (C2016 Deschamps FJ. This is an open-access article distributed under the terms of the Creative Commons Attribution License, which permits unrestricted use, distribution, and reproduction in any medium, provided the original author and source are credited. 